\title{
Novel Method to Study Stem Cells in Development and Disease
}

\author{
Austin Worden ${ }^{1}$, Matthew Stern ${ }^{2}$, Mark Uline ${ }^{3}$ and Jay Potts ${ }^{1}$ \\ ${ }^{1}$ University of South Carolina School of Medicine, Columbia, South Carolina, United States, ${ }^{2}$ Winthrop \\ University, Rock Hill, South Carolina, United States, ${ }^{3}$ University of South Carolina, Columbia, South \\ Carolina, United States
}

The long-standing, two-dimensional (2D) culture system that has allowed for cell functionality testing has begun to be replaced by more advantageous three-dimensional (3D) systems which provide a more accurate physiological response (i.e. in vivo tissue formation, increased differentiation, reduced proliferation, etc) [1]. One such 3D culture technology that allows for a tunable extracellular matrix (ECM) is the collagen hydrogel. Collagen hydrogels have aided in the advancements of drug delivery, tissue engineering and repair, and cellular processes such as cell migration and wound healing. The standard use of collagen gels in various ways has been to embed the cells within the collagen gel and allow gel contraction and treat the cells or gel in various ways and observe contraction. We have used a new method to produce a novel physiological response when we cultured adipose derived stem cells (ADSCs) on top of a Collagen I hydrogel. When place on the surface of the gel, the ADSCs migrate to form a ring-like structure, we have termed a toroid. These toroids can be thought of as self-organizing tissue structures [2]. Toroids are never formed when cells are embedded within the collagen gel. Interestingly, the developing toroid mimics the shape of the well in which it is formed (i.e. circle, square, rectangle, etc). Previously, our lab discovered that CXCL12, using a gene targeted analysis through Nanostring Technologies, was upregulated during toroid formation when compared to cells embedded in the gel. CXCL12 is a chemokine that is expressed in several tissues including the brain, heart, bone marrow. Its cognate receptor is CXCR4 and when bound induces a number of different signaling pathways which have been implicated in stem cell mhoning, angiogenesis, neural development, and diseases such as HIV, and cancer.

Our recent studies expand on the above finding by modeling cell-cell and cell-ECM interactions in single- and multi-cellular environments as well as quantifying the molecular mechanisms and remodeling occurring during toroid formation. While the majority of our experimentation uses PureCol Collagen I, we have tested no less than 9 formulations of matrices including three different concentrations of Collagen I (PureCol, Nutrigen, and Fibricol), Collagen III, Collagen V, Rat Tail Collagen, and three types of synthetic matrices (AlphaBioGel, Matrigel, and VitroGel3D) and used no less than 10 cell types including several types of stem cells, cancer cells, cardiac fibroblasts (NHFs), and microvascular endothelial cells.

Figure 1 shows the resulting multi-cell toroid. Mouse ADSCs, (red) and mouse NHFs, (green) were mixed prior to culturing them to produce a toroid. Interestingly, they appeared to mix, migrate, and form a standard toroid. There appeared to be no overlapping signal suggesting a communication that could be via gap junctions or microvilli [3]. We have begun to examine the importance of the CXCL12/CXCR4 pathway which was found to be upregulated during toroid formation. To test this, ADSCs were cultured to form toroids and embedded in Collagen I hydrogels. Gels were labeled using an antibody to CXCL12 and imaged on a Zeiss LSM 510 confocal microscope. Figure 2a shows a toroid after the standard 24-hours of incubation stained with CXCL12. We see strong CXCL12 expression (red, f-actin counterstained with Phalloidin 488-green) as cells migrate to form the toroid. However, there is little CXCL12 expression observed in the gels where cells were mixed in. This supports our transcriptional data and suggests that CXCL12 is likely important in toroid formation.

To summarize, we have created a novel method to examine cellular differentiation and resembles processes found in development and disease. This method creates toroids which appear universal in creation with the caveat that cancer cells do not form toroids. Our system allows for tunability and reproducibility to investigate 
stem cell interactions and programming. By using this new culture model we are uniquely positioned to explore (1) the understanding of early stem cell development, (2) the role of CXCL12 signaling in toroid formation, and (3) a novel system suitable for creating disease treatments using stem cells [4].

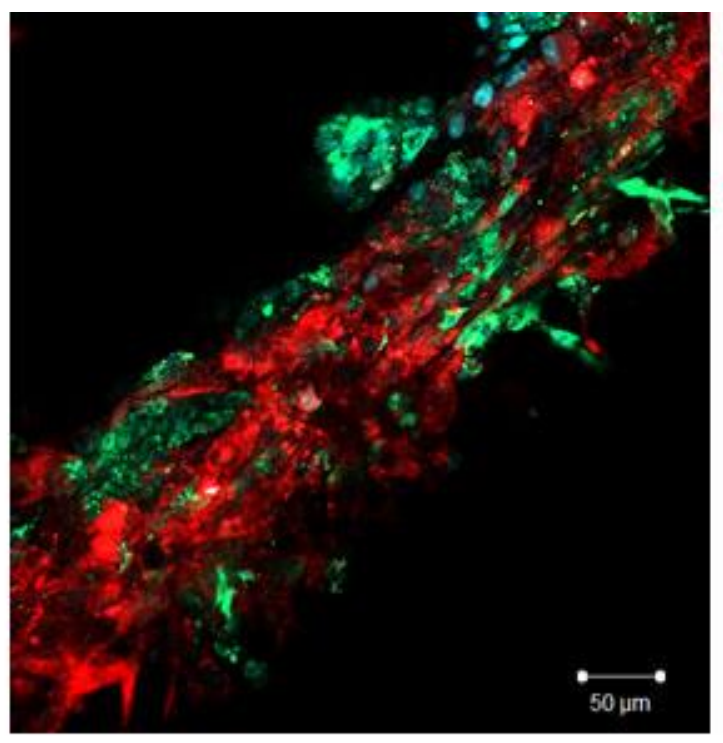

Figure 1. The toroid formed by culturing two different types of cells (ADSCs, NHF) on the surface of a collagen hydrogel. The two cell types have intermingled within the toroid structure. Scale bar $=50 \mu \mathrm{m}$, Red $=$ ADSC, Green=NHF, Blue $=$ DAPI

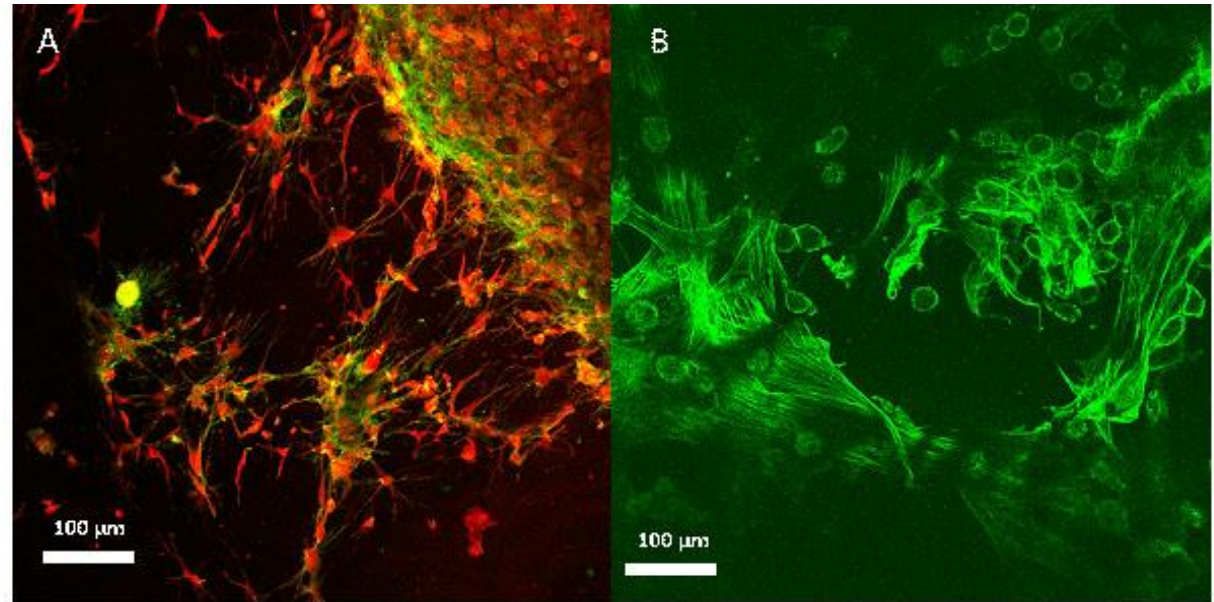

Figure 2. Confocal images of the Toroid versus mixed in hydrogels stained with CXCL12. A) Cells placed on top of a hydrogel form toroids with strong CXCL12 expression. B) Cells embedded into the hydrogel do not form toroids and show little to no CXCL12 expression. Scale bars $=100 \mu \mathrm{m}$, Green-Phalloidin, Red-CXCL12

\section{References}

[1] Kapałczyńska M. et al, Arch Med Sci 14 (2018) p. 910.

[2] Potts JD. et al, Microsc Microanal 24 (2018) p. 1362.

[3] Ali Khawar I. et al, Neoplasia 20 (2018) p. 800.

[4] The authors acknowledge funding from SPARC graduate research grant, Cook Biotech, FirstString Research Inc, NIH 2 P20-RR016434-06, NIH INBRE grant for South Carolina P20GM103499, R01 HL126747 